\title{
A Determination of the Central Charge at the Crumpling Transition
}

\author{
B. Jegerlehner ${ }^{a}$ and B. Petersson ${ }^{a}$ \\ ${ }^{2}$ Fakultät für Physik, Universität Bielefeld, P.O. Box 100131, 33501 Bielefeld, Germany
}

We determine the central charge at the critical coupling of the crumpling transition in crystalline surfaces. We make two independent determinations using periodic and free boundary conditions on a strip. The results are consistent, and the most precise value coming from the periodic lattice is $c=-0.10(22)$. The determination of the critical coupling gives also. through finite size scaling, values for the critical exponents $\nu=0.60(20)$ and $\alpha / \nu=1.11(11)$.

\section{INTRODUCTION}

Considerable efforts have been made in the last years in order to obtain a welldefined string theory in non critical dimensions. Dynamically triangulated random surfaces with a term that suppresses external curvature is an interesting model. which may have a critical point where a string theory could be defined also for $d>1$. A one loop calculation in the continuum. as well as calculations in the limit of infinite embedding dimension indicate the absence of such a transition. [1] [2] However, lattice calculations indicate the precense of a transition for a finite value of of the extrinsic curvature coupling $\lambda .[3]$ A recent calculation gives strong numerical evidence that the string tension goes to zero at this point. and scales consistently with the mass gap. [4] The above model should be an example of two dimensional gravitation coupled to a two dimensional matter fields. Naively, the embedding dimension $d$ corresponds to the number of degrees of freedom of these matter fields. In the present work we study the underlying model on a fixed flat triangulation, corresponding to the underlying two dimensional field theory not yet coupled to gravitation. We have chosen the embedding dimension $d=3$. This choice is motivated by the equivalence of the model to a statistical mechanics model of biological membranes or crystalline surfaces. Earlier numerical studies of the model can be found in [5][6][1], and references therein.

\footnotetext{
*Present address: Max-Planck-Lnstilut für Plysik. Foehringer Ring 5. 80805 Mündhen, Ciermany.
}

In this article we report on a new precise measurement of the central charge $c$, which should give the actual effective number of degrees of freedom. In order to obtain a precise measurement of the quantity $c$. we need to determine precisely the critical point. As a byproduct of this measurement we obtain through finite size scaling the critical exponents $a$ and $\nu$, which can be compared to earlier numerical calculations. as well as to analytical calculations in the large d limit. [8]

\section{THE MODEL}

The action is given by

$S=S_{y}+S_{c}$

where

$S_{y}=\frac{1}{2} \sum_{\langle i, j\rangle}\left(\vec{I}_{i}-\vec{I}_{j}\right)^{2}$

and

$S_{c}=\lambda \sum_{\left\langle\Delta_{1}, \Delta_{j}\right\rangle}\left(1-\vec{n}_{i} \cdot \vec{n}_{j}\right)$

Ilere $\bar{I}_{i}$ is a three dimensional vector at the site $i$ of the two dimensional lattice giving the coordinates of the site in the embedding space. The expression $\langle i . j\rangle$ denotes nearest neighbours. The expression $\left\langle\Delta_{i}, \Delta_{3}>\right.$ denote neighbouring triangles. sharing a link, and $\vec{n}_{i}$ the normal to the triangle $\lambda_{i}$. The triangulation is fixed and regular. 
The partition function is given by

$Z=\int \prod_{i=1}^{N} d \bar{X}_{i} \delta\left(\sum_{i} \bar{X}_{i}\right) e^{-S\left(\hat{X}^{2}\right)}$

The delta function takes care of the trivial center of mass translation. We define the specific heat by

$C=\frac{1}{N}\left(<S_{c}^{2}>-<S_{c}>^{2}\right)$

where $N$ is the number of vertices.

For the determination of the central charge we use the finite size formula for a $L \times M$ strip in the limit where $M$ is very large. We choose it as a constant much larger than the values used for L. One then has

$\frac{F}{L M}=a+\frac{b}{L}-\frac{2}{\sqrt{3}} \frac{\pi}{x} \frac{c_{\text {eff }}}{L^{2}}$

where $x=6$ for periodic and $x=32$ for free boundary conditions. The factor $2 / \sqrt{3}$ is necessary because we measure distance along the side of the triangles. If the theory is unitary and conformally invariant, $c_{e f f}=c$ at the critical point. where $c$ is the central charge of the conformal field theory. The free energy $F$ is defined by

$F=-\log Z$

With Monte Carlo techniques $\mathrm{F}$ can be found up to a constant from the numerical integration of the relation

$\lambda \frac{d F}{d \lambda}=<S_{c}>$

At $\lambda=0$ the value of cess is known analytically to be equal to three. This fact and a numerical integration of $E_{q}$. (8) is thus sufficient to determine $c_{e} \int f$ for all $\lambda$ and in particular at the critical point $\lambda=\lambda_{c}$

\section{SIMULATION AND RESULTS}

The simulation algorithm consists of a metropolis step for the curvature part and metropolis, heatbath and overrelaxation steps for the gaussian part of the action. which improves the autocorrelation time by a factor of at lcast two for the lattices simulated.

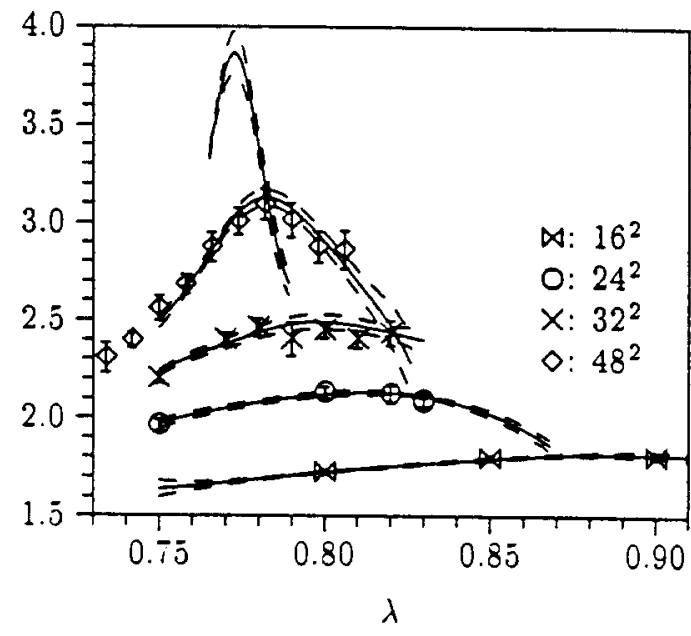

Figure 1. The specific heat as a function of lambda. The lines are obtained through the reweighting method.

The program runs on the Intel IPSC/860 Hypercube with 32 nodes and the Intel Paragon XP with 64 nodes. We parallelised the program over systems. Other parallelizations were less efficient.

The amount of data collected for the measurements of the specific heat for a determination of the critical coupling was about $10^{7}$ sweeps for each lattice size. For the largest lattice the autocorrelation time for the quantities measured is at most 15000 sweeps. We employ $5 \tau_{\text {int }}$ sweeps for thermalization. For the strips we used $6 \times 10^{6}$ sweeps for each data point and lattice sizes of $4 \times 100$ to $20 \times 100$.

The data were evaluated using the reweighting method. The criterion for the choice of the runs was. that the energy histograms should have an overlap of at least $10 \%$.

In Fig. 1 is shown the specific heat as a function of $\lambda$, for various lattice sizes $. V=L^{2}$. For the determination of the critical exponents and the critical coupling we rely on finite size scaling, predicting in leading order

$C_{\max }(L)=A+L^{\alpha / \nu}$
$\lambda_{\max }(L)-\lambda_{:}=\left(L^{-1 / \nu}\right.$.

The crucial quantities. $C_{\max }$ and $\lambda_{\max }$ were eval- 


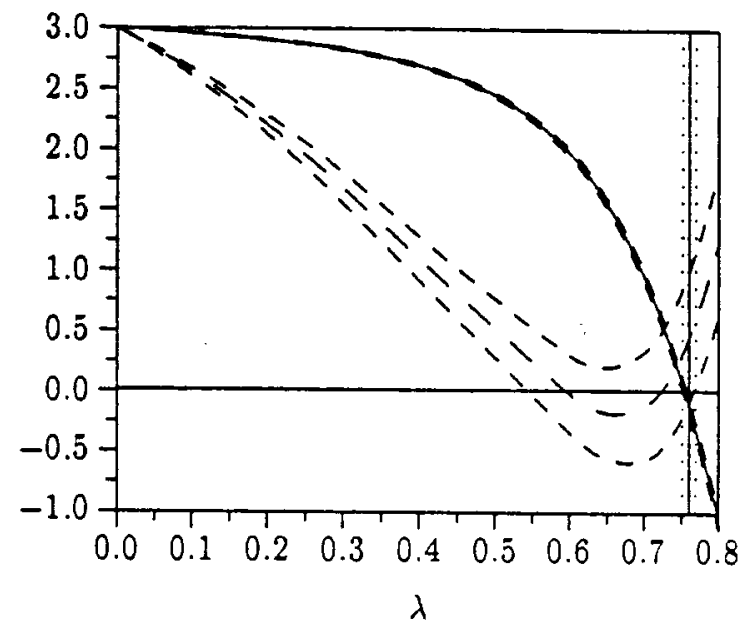

Figure 2. The effective central charge as a function of $\lambda$ for periodic (full line) and free (dashed line) boundary conditions. The vertical line gives the position of $\lambda_{c}$.

uated using the reweighting method. This give the following estimates:

$$
\begin{aligned}
\alpha / \nu & =1.11(11) \\
\nu & =0.60(20) \\
\lambda_{c} & =0.7605(90) .
\end{aligned}
$$

The values for $\alpha$ and $\nu$ are in agreement with Ref.[7]. At large d, on the contrary one has to leading order $\alpha=-d$ and $\nu=d / 2 .[8]$ This is not in agreement with our results. which are at $d=3$.

The main result of our work is a new measurement of the central charge at the critical point, which we determined above. For the determination of $c_{e f f}$ we used the formula (8) inserting formula (6) and also higher corrections to this formula. To take into account possible systematic effects, we performed the measurements both for periodic and free boundary conditions. The results are shown in Fig. 2. As one can see, the results of the two measurements are in good agreement with each other at the critical point. For free boundary conditions we have a dominating contribution from the surface term (the second term on the right hand side of ec. (6)), which is not present for periodic boundary conditions. Therefore the errors are considerably larger. We estinnate

$c_{e f f}=-0.10(22)$ for per. $b c$

$c_{e f f}=0.43(70)$ for free $b c$

The more precise value is not in agreement with a unitary, minimal conformally invariant model which would demand $c \geq 1 / 2$. Furthermore both values are definitely not in agreement with the analytic result at large $d$, namely

$c_{e j}=2 d$.

Our results show that the model studied here does not correspond to a conformal theory as the basis for a string theory in $d>1$. For the further understanding of the model as a theory of membranes. it is important to extend also the anaIytic calculations at large $d$, to get better agreement with our quite precise numerical data. Finally, our result for $c_{e} / f$ are in contradiction to the (unproven) hypothesis that the Zamolodition c-theorem can be applied to this model. (9] The latter theorem would demand $c_{\text {eff }} \geq d$.

Achnowledgements: We are very grateful to the HLRZ and ZA.H. Juelich for generous help and computer time. B. P. also thanks J. Ambjorn and F. David for helpful discussions.

\section{REFERENCES}

1. A. Polyakor, Nucl. Phys. B268 (1986) 406

2. F. David. Europhys. Lett. 2 (1986) $57 t$

3. S. Catterall. Phys. Lett. 220B (1989) 207

4. J. Ambjorn. A. Irbäck. J. Jurkiewicz and B. Petersson. Phys. Lett. 27.5B (1992) 29.5

j. J. B. Kogut and R. L. Renken, Nucl. Phys. B:348 (1991) 580

6. R. G. Harnish and J. F. Wheater. Nucl. Phys. B3.50 (1991) 861

†. J. F. Wheater and P. W. Stephenson, Oxford preprint OUTP-92-40P.

8. F. David and E. Ciuitter, Europhys. Lett. 5 (1988) 709

9. A. B. Zanolodchikov, Pisma Zh. Eksp. Teor. Fiz. 13. No $12(1086) 565$ 